\title{
Association Between Anthropometric Factors and Balance Among Elderly Fallers and Non-Fallers: A Cross-Sectional Study
}

\author{
Pothiraj Pitchai \\ MGM College of Physiotherapy, Navi Mumbai, pothirajpt@gmail.com \\ Reshma Gurav \\ MGM College of Physiotherapy, Navi Mumbai, reshmagurav81@gmail.com \\ Srishti Kuldeep Chauhan \\ MGM College of Physiotherapy, Navi Mumbai, srishti.1003@gmail.com
}

Follow this and additional works at: https://nsuworks.nova.edu/ijahsp

Part of the Medicine and Health Sciences Commons

\section{Recommended Citation}

Pitchai P, Gurav R, Chauhan SK. Association Between Anthropometric Factors and Balance Among Elderly Fallers and Non-Fallers: A Cross-Sectional Study. The Internet Journal of Allied Health Sciences and Practice. 2021 Jan 01;19(3), Article 7.

This Manuscript is brought to you for free and open access by the College of Health Care Sciences at NSUWorks. It has been accepted for inclusion in Internet Journal of Allied Health Sciences and Practice by an authorized editor of NSUWorks. For more information, please contact nsuworks@nova.edu. 


\title{
Association Between Anthropometric Factors and Balance Among Elderly Fallers and Non-Fallers: A Cross-Sectional Study
}

\begin{abstract}
Purpose: Falls are an emerging public health problem causing a cascade of medical, functional, and socioeconomic consequences. Apart from other widely explored risk factors affecting balance, anthropometric factors are also known to have an impact on balance. However, this relationship hasn't been studied extensively in older adults. This study aimed to evaluate the relationship between the anthropometric factors such as Body Mass Index (BMI), Body Fat Mass (BFM), Waist-Hip Ratio (WHR), Lower Limb Length (LLL), Foot Length (FL) and balance in the elderly among fallers and non-fallers. Method: This cross-sectional study was performed on 100 fallers and 100 non-fallers, aged 60 years and above. These participants were recruited by a stratified random sampling technique from Navi Mumbai region. All the above anthropometric factors were measured and recorded. Each participant's balance was assessed using the Mini-BESTest scale. Obtained scores were analysed in SPSS software; descriptive statistics, Spearman correlation coefficient, and Z scores were applied. Results: A sample size of 100 non-fallers, $50 \%$ male and $50 \%$ females, participated in this study. Among those participants classified as "fallers," $56 \%$ were males and $44 \%$ were females. The mean age of the non-fallers was $66 \pm 5.01$ and the mean age of the fallers was $67.72 \pm 6.73$. In fallers, WHR showed good negative correlation ( $r=-.807)$, BFM as moderate $(r=-.577)$ and BMI as fair $(r=-.426)$ whereas in non-fallers, BMI showed moderate $(r=-.546)$ and fair negative correlation for both WHR $(r=-.303)$ and BFM $(r=-.441)$. However, LLL and FL in both groups show little or no correlation. The Association of all anthropometric factors with the balance between fallers and non-fallers showed no-significant difference. It may be inter-group variance for age, gender and $\mathrm{BMI}$, as participants were not matched for these variables during the recruiting phase. Additionally, the reason for the fall was not explored, thus adding to the limitations of our study. Conclusion:This study demonstrated the impact of increased WHR, BFM and BMI on balance in the elderly fallers and non-fallers. Thus, it is important to screen these factors while assessing biological risk factors for predicting falls. This study further recommends exploring the normative value for anthropometric factors in a healthy elderly population.
\end{abstract}

\section{Author Bio(s)}

Reshma Gurav, MPTH in Musculoskeletal Physiotherapy. Former Associate Professor, Department of Musculoskeletal Physiotherapy, MGM College of Physiotherapy, Maharashtra University of Health Sciences, Navi Mumbai.India.

Srishti Kuldeep Chauhan, Former Intern, Department of Community Physiotherapy, MGM College of Physiotherapy, Maharashtra University of Health Sciences, Navi Mumbai.India

\section{Acknowledgements}

We acknowledge all the participants who participated in this study. Our Principal who encouraged and gave us permission to conduct this study. 


\title{
1IJAHSP \\ The Internet Journal of Allied Health Sciences and Practice \\ Dedicated to allied health professional practice and education \\ Vol. 19 No. 3 ISSN 1540-580X
}

\section{Association between Anthropometric Factors and Balance among Elderly Fallers and Non-Fallers: A cross sectional study}

\author{
Pothiraj Pitchai \\ Srishti Kuldeep Chauhan \\ Reshma Gurav \\ MGM College of Physiotherapy, Navi Mumbai \\ India
}

\begin{abstract}
Purpose: Falls are an emerging public health problem causing a cascade of medical, functional, and socio-economic consequences. Apart from other widely explored risk factors affecting balance, anthropometric factors are also known to have an impact on balance. However, this relationship hasn't been studied extensively in older adults. This study aimed to evaluate the relationship between the anthropometric factors such as Body Mass Index (BMI), Body Fat Mass (BFM), Waist-Hip Ratio (WHR), Lower Limb Length (LLL), Foot Length (FL) and balance in the elderly among fallers and non-fallers. Method: This cross-sectional study was performed on 100 fallers and 100 non-fallers, aged 60 years and above. These participants were recruited by a stratified random sampling technique from Navi Mumbai region. All the above anthropometric factors were measured and recorded. Each participant's balance was assessed using the Mini-BESTest scale. Obtained scores were analysed in SPSS software; descriptive statistics, Spearman correlation coefficient, and Z scores were applied. Results: A sample size of 100 non-fallers, $50 \%$ male and $50 \%$ females, participated in this study. Among those participants classified as "fallers," $56 \%$ were males and $44 \%$ were females. The mean age of the non-fallers was $66 \pm 5.01$ and the mean age of the fallers was $67.72 \pm 6.73$. In fallers, WHR showed good negative correlation ( $r=-.807), \mathrm{BFM}$ as moderate $(r=-.577)$ and BMl as fair $(r=-.426)$ whereas in non-fallers, BMl showed moderate $(r=-.546)$ and fair negative correlation for both WHR ( $r=-.303)$ and BFM ( $r=-.441)$. However, LLL and FL in both groups show little or no correlation. The Association of all anthropometric factors with the balance between fallers and non-fallers showed nosignificant difference. It may be inter-group variance for age, gender and BMI, as participants were not matched for these variables during the recruiting phase. Additionally, the reason for the fall was not explored, thus adding to the limitations of our study. Conclusion: This study demonstrated the impact of increased WHR, BFM and BMI on balance in the elderly fallers and nonfallers. Thus, it is important to screen these factors while assessing biological risk factors for predicting falls. This study further recommends exploring the normative value for anthropometric factors in a healthy elderly population.
\end{abstract}

Keywords: Anthropometry, Balance, Fallers, Non-fallers, older adults. 


\section{INTRODUCTION}

Falls are an emerging public health problem and a barrier to being physically active, not only in India, but across the globe. Falls are a growing concern among the elderly and are known to threaten their independence, causing a cascade of medical and socioeconomic consequences. The incidence of falls varies among countries; however, the frequency of falls increases above the age of 60 years along with an increase in frailty. ${ }^{1}$ In India, the prevalence of falls among the elderly aged 60 years and above is $14 \%$ to $53 \%$ and reported to be highest among women as well as institutionalised older adults. ${ }^{2-6}$ The consequences of falls vary from mild to severe injuries such as bruises, lacerations, fractures, traumatic brain injuries, and fatality in severe cases. The long-term repercussions include decreased physical function, fear of falling, and institutionalisation. ${ }^{1}$ Incidentally, falls are reported to be the leading cause of death in the elderly.

There is an urgent need for coordinated and collaborative efforts from health professionals, researchers, policymakers, and healthcare delivery systems to prevent falls. Fall prevention must be emphasized in public health policies and health programmes for elderly people. The World Health Organization's(WHO) 2007 global report on fall prevention in older age has classified risk factors for falls into four main categories: biological, environmental, behavioural, and socio-economic factors. Biological risk factors can be intrinsic and extrinsic. Intrinsic risk factors include chronic diseases, age-related decline in the neuromusculoskeletal system, and a decline in the senses (vision, hearing, smell, taste). Extrinsic risk factors are physical environmental factors that include features in the home or community that may pose hazards, such as slippery or uneven surfaces, improper steps, staircases without proper railings, poor building design, and insufficient lighting. ${ }^{1}$ Studies have found that extrinsic factors are more likely to be the cause of falls among the 60 to 74 years age group, whereas intrinsic factors are more likely to be the cause for those aged 75 years and older. ${ }^{7}$

Because of an increased life expectancy of the elderly in developed as well as developing countries, it is imperative to shift the focus to preventive strategies for reducing the incidence of falls in the elderly. Such a shift will not only reduce the incidence of falls, but may also decrease the socioeconomic burden. To date, several studies have been conducted not only to examine the effectiveness of fall preventive programs but also multimodal and interdisciplinary preventive approaches. However, current literature lacks a comprehensive risk factor assessment tool for falls. Identifying the factors that threaten balance can help to improve the accuracy of diagnosis and quality of treatment, which forms the foundation for preventing falls.

Balance is an eminent factor that should be evaluated while screening for the probability of falls in the elderly. Balance deficits in the elderly can be assessed by choosing from a variety of assessment instruments that exist, including simple observations, clinical tests, posturographic measurements, force platforms, motion analysis, and functional scales such as Berg's Balance scale, Tinetti

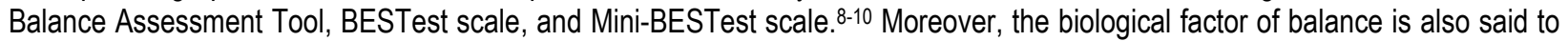
be influenced by anthropometric factors such as body weight, height, Body Mass Index(BMI), Body Fat Mass(BFM)and Lower Limb Length(LLL). ${ }^{11,12}$ Interestingly, the literature also reported that variation in the leg length and foot length (FL) positively correlates with posture thus indirectly influencing on balance. ${ }^{13}$ Multiple studies on various populations have demonstrated that an increase in body mass can change the antero-posterior position of the center of gravity (COG). Likewise, an individual limb length can alter the vertical height of COG, whereas the foot length determines the flexibility of $C O G$ and therefore influences postural stability. $11,13,14$

Despite the availability of the exitant balance tools that assess various factors related to balance, none include the assessment of anthropometric factors. While the relationship between anthropometric factors and postural balance has been explored in young adults in the past, this relationship has not been as widely explored for older adults. ${ }^{15}$ Can these anthropometric factors also detect postural balance deficits in the elderly to predict the risk of falls? This study aims to find out the association between anthropometric factors and balance among elderly fallers and non-fallers with the primary objective to measure anthropometric factors such as BMI, BFM, Waist-Hip Ratio(WHR), LLL, and FL.

\section{METHODS}

\section{Participants}

This is a cross-sectional study conducted between July 2018 to December 2018 at the physiotherapy outpatient department of MGM Hospital, Navi Mumbai, and old-age homes from the institutional field practice area of Navi Mumbai. The institutional research review committee approved this study(Ref No. MGM/COP/IRRC/125/2018). The purpose of this study was explained and informed consent was obtained from all the participants. Individuals above the age of 60 , both male and female gender, without any history of falls were placed in the non-fallers group, while those with a minimum of two falls in the past six months were placed in the fallers group. 
Individuals with visual impairments like cataracts, macular degeneration, anddepth perception difficulties were excluded. Subjects with pre-existing neurological conditions such as Parkinson's, cerebral vascular accident, peripheral neuropathy, vestibular disorders and impaired cognitive function were also excluded. Those with musculoskeletal conditions and impairments affecting lower limb such as arthritis, kyphosis, scoliosis, foot disorders, lower limb amputation, surgery involving lower quarter, lower body muscle weakness, and those with traumatic falls were carefully excluded from the study.

A fall was defined as any unintentional change in position where the person ends up on the floor, ground, or other lower-level location excluding intentional change in position to rest. ${ }^{1}$ History of fall in the past 6 months was screened by a self-made structured questionnaire. Participants who reported more than one fall including a slip or trip in which participants lost their balance and landed on the floor or ground or a lower level was grouped into the fallers group and those who had no history of fall were grouped into a non-fallers group.

Participants were recruited through a stratified simple random sampling technique. A survey was conducted in the old age home. Moreover, patients and their caregivers visiting the physiotherapy outpatient department were assessed for eligibility. Out of eligible fallers and non-fallers who satisfied inclusion and exclusion criteria, 100 were recruited from each group through a computergenerated simple random number and subjected for anthropometric measurement.

The sample size was calculated by using OpenEpi online software, version 3.01. Considering $24.98 \%$ of prevalence of falls in elderly, a $95 \%$ confidence level(1-alpha), power set at $80 \%$, the ratio of controls to cases as1, the proportion of controls with exposure as 45 , and proportion of cases with exposure as 25 , the total required sample was $200--100$ each in faller group and non-faller group. ${ }^{2}$

\section{Measurement of Anthropometric Factors}

Anthropometric measurements such as BMI and WHR for the study participants were in accordance with the National Health And Nutrition Examination Survey. ${ }^{16} \mathrm{~A}$ wall-mounted stadiometer was used to measure the height within 0.1 centimetres. The subject was asked to remove their footwear and stand against the wall, heels together, hands at the side of the body, palms facing the thigh, head in Frankfurt plane, and the horizontal limb of the stature meter firmly placed on the top of the head to obtain a reading. Weight was recorded by a calibrated, portable digital weighing scale with a range of 150 kilograms. The accuracy of measuring weight load in the scale was confirmed by using test loads of $10 \mathrm{~kg}, 25 \mathrm{~kg}, 50 \mathrm{~kg}, 75 \mathrm{~kg}, 100 \mathrm{~kg}, 110 \mathrm{~kg}$ and $150 \mathrm{~kg}$. Participants were given scrubs to wear and instructed to remove footwear before stepping on the weighing scale. BMI was calculated using the formula where weight in kilograms was divided by the square of height in meters. According to WHO BMI classification, the calculated BMI was categorised into underweight $\left(\mathrm{BMI}<18.5 \mathrm{~kg} / \mathrm{m}^{2}\right)$, normal weight $\left(18.5-24.9 \mathrm{~kg} / \mathrm{m}^{2}\right)$, overweight $(25.0-29.9$ $\left.\mathrm{kg} / \mathrm{m}^{2}\right)$, class I obesity $\left(30.0-34.9 \mathrm{~kg} / \mathrm{m}^{2}\right)$, class II obesity $\left(35.0-39.9 \mathrm{~kg} / \mathrm{m}^{2}\right)$ and class III obesity $\left(\geq 40.0 \mathrm{~kg} / \mathrm{m}^{2}\right)$.

Circumference of the waist was measured in centimetres by placing the measuring tape in a horizontal plane around the abdomen at the measurement site, on the right side of the trunk. The measurement site was marked by palpating the hip area to locate the right ilium of the pelvis; a horizontal line was drawn just above the uppermost lateral border of the right ilium and crossed this mark at the midaxillary line. The tape was positioned parallel to the floor, snugly without compressing the skin and underlying subcutaneous tissue. Participants were asked to relax their abdomen, and at the end of normal expiration, a single measurement was recorded in centimetres to the nearest millimetre. Hip circumference was also recorded in centimetres, using the same technique, but the measuring tape was placed around the widest part of the hips or buttocks. The waist-hip ratio was then calculated by dividing the waist circumference by the hip circumference.

Lower limb length was measured by a direct clinical method using a measuring tape. ${ }^{17,18}$ True limb length was measured after squaring the pelvis. The length from the anterior superior iliac spine to the tip of medial malleolus was noted while both lower limbs were resting in identical supine anatomical position. Foot length was measured by measuring the distance between the tip of the hallux or the tip of the second toe when the second toe was longer than the hallux to the most prominent point of the back of the heel. The mean of both feet was calculated and used for statistical analysis. ${ }^{13}$

Skinfold thickness was measured using a calibrated, standard skinfold caliper. It was measured at the non-dominant site of triceps, abdomen, and suprailiac areas, after carefully marking the appropriate anatomical landmarks. ${ }^{16} \mathrm{~A}$ minimum of two measurements at the sites were taken and if the two measurements at a site differed by more than $3 \mathrm{~mm}$ a third measurement was taken. The mean of the two closest measures was calculated and used in the calculation of body fat by using the three site formula. ${ }^{19}$

Participants' balance was measured using the Mini-BESTest scale which consist of 14 items and broadly evaluates performance tasks based on anticipatory postural adjustments, postural responses, sensory orientation, and dynamic gait. Each of the items 
was scored on a 3-point ordinal scale ranging from 0 to 2 . The maximum possible total score is 28 , with a higher score indicating better balance. ${ }^{20}$

\section{Statistical Analysis}

Gathered data was entered and analysed using SPSS statistical software version 17 (SPSS Inc. Chicago, IL, USA). For qualitative variables, descriptive statistics, such as frequency, percentage, mean, and standard deviation, were applied. The probability of normality of the study variables was checked with the Kolmogorov-Smirnov test. Since the data did not follow a normal distribution, the non-parametric test was applied. The relationship between balance scores and anthropometric factors was calculated with the Spearman correlation coefficient. The strength of the correlation was considered between -1.00 to 1.00 . The correlation was categorised as good when $r=$ above 0.75 , moderate as $r=0.50$ to 0.75 , fair as $r=0.25$ to 0.50 and little or no relationship as $r=0.00$ to 0.25 . To find out the statistical difference between the correlation coefficient of two groups of fallers and non-fallers, the $Z$ score was calculated by using log 10 of values. A Z obs value between -1.96 and +1.96 is considered as statistically non-significant.

\section{RESULTS}

\section{Clinical Characteristics of the Participants}

A total of 1243 participants were screened for eligibility. One hundred (100) participants were assigned to each faller and non-faller group. The participant recruitment strategy is shown in the flow diagram in Figure 1. In a sample space of 100 non-fallers, $50 \%$ were males and $50 \%$ were females. In fallers, $56 \%$ were males and $44 \%$ were females. The summary of the descriptive statistics of the study participants is provided in Table 1. According to BMI categories in non-fallers, $14 \%$ demonstrated normal BMI, $50 \%$ for excess weight and $36 \%$ fell into the obese category. However, among fallers, $3 \%$ had normal BMI, $22 \%$ fell into the excess weight category and $75 \%$ were obese.

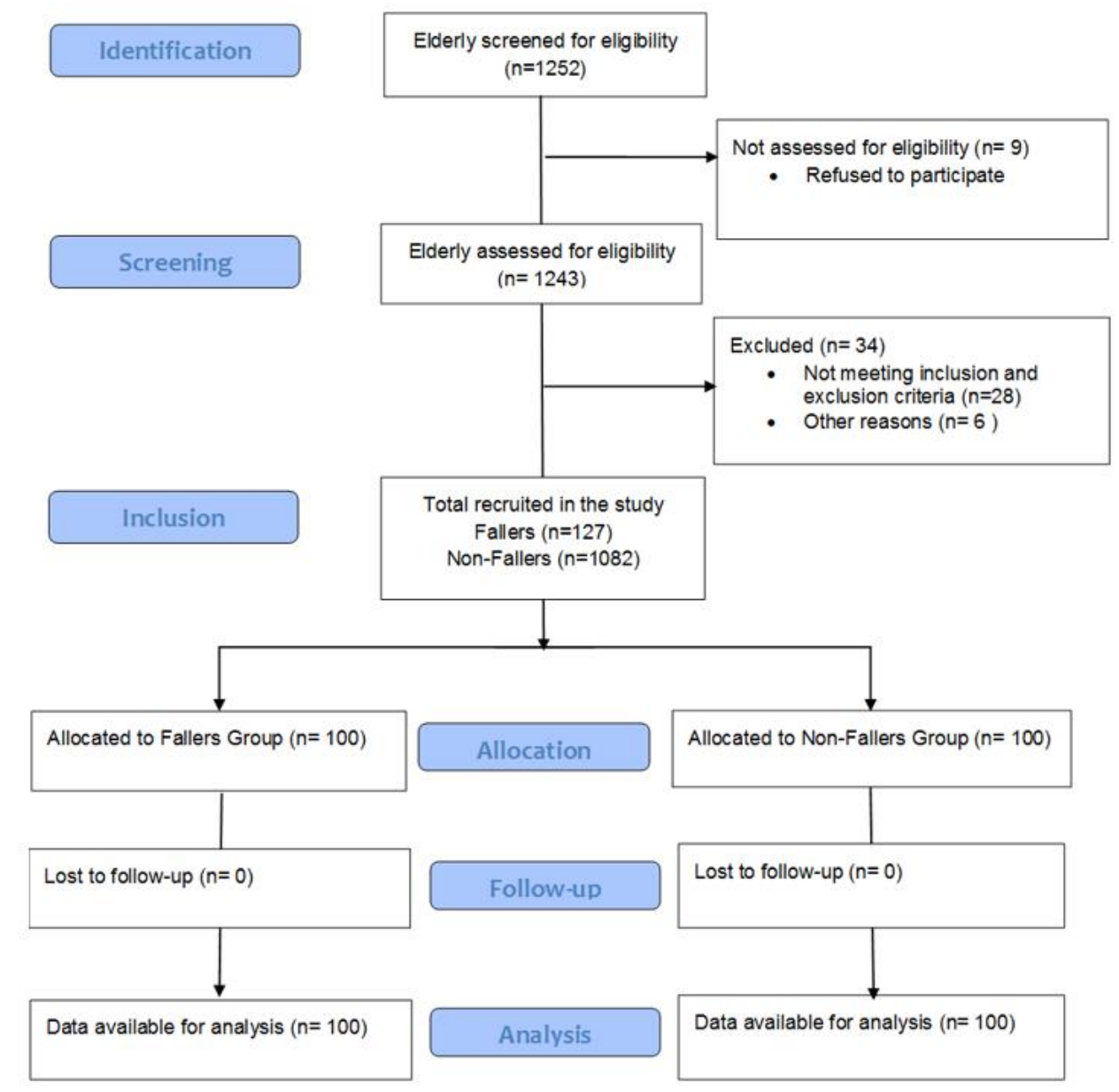

Figure 1. Flow diagram of participant recruitment 
Table 1. Descriptive statistics of the study participants.

\begin{tabular}{|c|c|c|c|c|c|c|}
\hline \multicolumn{3}{|c|}{ Non-Fallers $(n=100)$} & \multirow{3}{*}{ Parameter } & \multicolumn{3}{|c|}{ Fallers $(n=100)$} \\
\hline \multicolumn{2}{|l|}{ Range } & \multirow{2}{*}{ Mean $\pm S D^{*}$} & & \multirow{2}{*}{ Mean $\pm S D^{*}$} & \multicolumn{2}{|l|}{ Range } \\
\hline Maximum & Minimum & & & & Minimum & Maximum \\
\hline 82 & 60 & $66 \pm 5.01$ & Age & $67.72 \pm 6.73$ & 60 & 84 \\
\hline 30.82 & 20.7 & $24.77 \pm 1.93$ & BMI & $27.2 \pm 2.92$ & 21.22 & 33.39 \\
\hline 1.34 & 0.723 & $0.95 \pm .10$ & Waist Hip Ratio & $1.11 \pm .21$ & 0.82 & 1.54 \\
\hline 93.2 & 70.3 & $86.04 \pm 4.82$ & Lower Limb Length & $83.2 \pm 8.17$ & 65.9 & 97.7 \\
\hline 28.2 & 15.6 & $21.95 \pm 2.58$ & Foot Length & $21.27 \pm 2.76$ & 16.2 & 27.3 \\
\hline 0.9 & 9.3 & $16.65 \pm 4.26$ & Body Fat Mass & $21.77 \pm 5.06$ & 13.4 & 34.9 \\
\hline 26 & 10 & $19.1 \pm 3.52$ & Mini BESTest & $10.11 \pm 2.74$ & 7 & 18 \\
\hline
\end{tabular}

${ }^{*}$ Data are presented as mean \pm standard deviation.

\section{Correlation Between Anthropometric Variables and Balance}

The correlation between anthropometric factors and balance measured by Spearman's correlation coefficient between fallers and non-fallers are described in Table 2. There is a negative correlation in both the groups between balance and anthropometric factors such as BMI, WHR, and BFM, which are statistically significant as well. In fallers, WHR shows a good correlation, BFM shows a moderate correlation, and BMI shows a fair correlation. In non-fallers, BMI shows a moderate correlation, and both WHR and BFM show a fair correlation. However, LLL and FL in both groups show little or no correlation.

Table 2. Correlation between balance and anthropometric factors in fallers and non-fallers.

\begin{tabular}{|l|l|l|l|l|}
\hline P value & $\begin{array}{l}\text { 'r' Value } \\
\text { in } \\
\begin{array}{l}\text { Non- } \\
\text { Fallers } \\
(\mathbf{n}=100)\end{array}\end{array}$ & $\begin{array}{l}\text { Anthropometric } \\
\text { Factors }\end{array}$ & $\begin{array}{l}\text { 'r' Value } \\
\text { in } \\
\text { Fallers } \\
(\mathbf{n}=100)\end{array}$ & $P$ value \\
\hline .000 & $-.546^{* *}$ & BMl & $-.426^{* *}$ & .000 \\
\hline .002 & $-.303^{* *}$ & Waist Hip Ratio & $-.807^{* *}$ & .000 \\
\hline .000 & $-.441^{* *}$ & Body Fat Mass & $-.577^{* *}$ & .000 \\
\hline .386 & .088 & Lower Limb Length & -.047 & .644 \\
\hline .171 & .138 & Foot Length & .038 & .708 \\
\hline
\end{tabular}

**. Correlation is significant at the 0.01 level (2-tailed).

\section{Correlation Coefficient Between Fallers and Non-fallers}

The calculated $Z$ scores between anthropometric factors and balance among fallers and non-fallers show no statistical difference. The observed difference in the correlation noted between the two groups could have been due to chance. Details of these scores are summarised in Table 3.

Table 3. Association score for anthropometric factors between fallers and non-fallers

\begin{tabular}{|l|c|}
\hline $\begin{array}{l}\text { Anthropometric } \\
\text { Factors }\end{array}$ & $\begin{array}{c}\mathbf{Z} \\
\text { Score }\end{array}$ \\
\hline BMl & $-0.267^{*}$ \\
\hline Waist Hip Ratio & $0.399^{*}$ \\
\hline Body Fat Mass & $0.177^{*}$ \\
\hline Lower Limb Length & $0.129^{*}$ \\
\hline Foot Length & $0.004^{*}$ \\
\hline
\end{tabular}

${ }^{*}$ Correlation coefficient is not statistically significant.

\section{DISCUSSION}

This study assessed the relationship between anthropometric factors such as BMI, WHR, BFM, LLL, FL and balance among the elderly population of fallers and non-fallers. Results of this study revealed that increased BMI is associated with decreased balance 
in both groups. This reiterates findings from a previous study where younger adults with an increased BMI demonstrated worse postural response. ${ }^{15}$ The changes in body composition associated with normal aging cause an increase in the BMI of $1.5-2.5 \mathrm{~kg} / \mathrm{m} 2$ /decade in both men and women, which is negatively associated with the level of physical activity. On the other hand, increased BMI among fallers could be further associated with a decreased level of physical activity and functional impairment because of a fear of falling. In both cases, it has been observed as BMI increases, postural stability decreases, which further increases the risk of falls in older adults. ${ }^{21-24}$

This study also demonstrated an inverse relationship of WHR with balance among fallers and non-fallers. It is likely due to central fat distribution altering the center of mass and increasing the load on the hips, thus increasing sway in the mediolateral direction and affecting postural balance. Similarly, Alonso et al in 2012 explored the relationship between body composition and posturographic measurements in young adults. They have found that an increase in WHR results in poor postural balance with increased mediolateral sway velocity. ${ }^{25}$ The reason for higher BMI and waist-hip ratio in fallers as compared to non-fallers could vary from their difference in lifestyle to other health-related issues. Discussing these reasons is beyond the scope of this study. However, these high BMI and WHR values may have contributed to reducing the balance scores in our fallers and non-fallers.

The higher the body mass, the stronger the musculoskeletal force needed to overcome the body's inertia and maintain the balance against gravitational force when imbalanced. ${ }^{26}$ Thus, individuals with higher body mass require greater movement off the centre of gravity to maintain balance under unstable conditions. However, failing to generate these forces for the altered center of mass due to aging increases the risk of losing postural balance, hence increasing the risk of fall.26 The reciprocal relationship of body composition with balance was further illustrated in this study, where the increased body fat mass of both groups had a decreased balance response. Previous literature also reports that as body fat mass increases, the strength of the muscle decreases. Thus, individuals with higher body fat fail to generate sufficient muscle force and motor control necessary for maintaining postural control. ${ }^{25}$

The direct relationship between foot length and balance scores in this study suggests that individuals with longer foot length have better balance and lower risk of fall. It is plausible that longer feet increase the base of support area and thus provides a wider area for the COG to restore balance during instability. These findings are consistent with previous literature. 25,28 Likewise, fallers and non-fallers in this study developed an opposite association between lower limb length and balance. This negative correlation can be explained by the fact that longer lower limb length would increase the height of center of gravity(COG), causing the line of gravity to fall outside base of support and thus affecting one's balance

The findings of this study demonstrate an inverse relationship between BMI, WHR, BFM and balance. Those individuals with higher body weight and body fat composition have less control over their balance.. Evaluation of these anthropometric factors, therefore, helps identify the risk of falls in the elderly.

\section{Limitations}

The Association of all anthropometric factors with the balance between fallers and non-fallers showed no-significant difference. It may be inter-group variance for age, gender and BMI, as participants were not matched for these variables during the recruiting phase. Additionally, the reasons for the falls were not explored, thus adding to the limitation of our study. We believe more accurate data could have been obtained for body fat measurements through the latest available devices as opposed to the skin fold caliper used in this study. ${ }^{29}$

\section{CONCLUSION}

This study has demonstrated the impact of increased WHR, BFM and BMI on balance in the elderly fallers and non-fallers. It is imperative to include these anthropometric factors while screening biological risk factors for falls in the elderly. Integration of these body composition measures into the evaluation of the elderly can help to identify balance deficits and guide the adoption of appropriate strategies to prevent or manage the risk of falls. We further recommend obtaining the normative values for these anthropometric variables in a healthy elderly population, alongside exploring its associations with the risk of falls. 


\section{ETHICS APPROVAL AND CONSENT TO PARTICIPATE}

This study was approved by the Institutional research review committee of MGM College of Physiotherapy, Navi Mumbai, India.

\section{HUMAN AND ANIMAL RIGHTS}

Animals are not used in our study. Study conducted on humans were in accordance with the declaration of Helsinki and Indian Council of Medical Research Guideline, India.

\section{CONFLICT OF INTEREST}

The authors declare no conflict of interest, financial support or otherwise for this study.

\section{REFERENCES}

1. World Health Organization. WHO global report on falls prevention in older age. Available from: http://www.who.int/ageing/Publications/Falls_prevention7March.pdf.

2. Pitchai $P$, Dedhia HB, Bhandari N, Krishnan D, D'Souza NR, Bellara JM. Prevalence, risk factors, circumstances for falls and level of functional independence among geriatric population - A descriptive study. Indian J Public Health 2019; 63(1):21-6.

3. Dsouza SA, Shringapure A, Karol J. Circumstances and consequences of falls in Indian older adults. Indian J Occup Ther 2008; 4:3-11.

4. Johnson SJ. Frequency and nature of falls among older women in India. Asia Pac J Public Health 2006; 18(1):56-61.

5. Rubenstein LZ. Falls in older people: epidemiology, risk factors and strategies for prevention. Age Ageing 2006; 35 Suppl 2:ii37-ii41.

6. Scuffham $P$, Chaplin S, Legood R. Incidence and costs of unintentional falls in older people in the United Kingdom. $J$ Epidemiol Community Health 2003; 57(9):740-4.

7. Murphy S, Tickle-Degnen L. Participation in daily living tasks among older adults with fear of falling. Am J Occup Ther 2001; 55(5):538-44.

8. Di Carlo S, Bravini E, Vercelli S, Massazza G, Ferriero G The Mini-BESTest: a review of psychometric properties. Int J Rehabil Res.2016; 39(2):97-105.

9. Godi M, Franchignoni F, Caligari M, Giordano A, Turcato AM, Nardone A. Comparison of reliability, validity, and responsiveness of the mini-BESTest and Berg Balance Scale in patients with balance disorders. Phys Ther.2013; 93(2):158-67.

10. Yingyongyudha A, Saengsirisuwan V, Panichaporn W, Boonsinsukh R. The Mini-Balance Evaluation Systems Test (Mini-BESTest) Demonstrates Higher Accuracy in Identifying Older Adult Participants With History of Falls Than Do the BESTest, Berg Balance Scale, or Timed Up and Go Test. J Geriatr Phys Ther.2016; 39(2):64-70.

11. Molikova R, Bezdickova M, Langova K, Holibka, V David O, Michalikova Z, et al. The relationship between morphological indicators of human body and posture. Biomed Pap Med Fac Univ Palacky Olomouc Czech Repub 2006; 150(2):261-5.

12. Kejonen $\mathrm{P}$, Kauranen $\mathrm{K}$, Vaharanta $\mathrm{H}$. The relationship between anthropometric factors and body-balancing movements in postural balance. Arc Phys Med Rehabil 2003; 84(1):17-22.

13. Arora M. Prediction of Stature by the Measurement of Lower Limb Length and Foot Length. Int J Med Res Prof. 2016; 2(2); 303-05.

14. M. Prado, Stoffregen T. A., Duarte M. Postural sway during dual tasks in young and elderly adults. Gerontology 2007; 53(5):274-281.

15. D’Andréa Greve J. M., Cuğ M, Dülgeroğlu D., Carlos Brech G., Castilho Alonso A. Relationship between Anthropometric Factors, Gender, and Balance under Unstable Conditions in Young Adults. BioMed Research International 2013; 850424:1-5.

16. Center for Disease Control and Prevention. National Health and Nutrition Examination Survey; January 2007. Available from: https://www.cdc.gov/nchs/data/nhanes/nhanes_07_08/manual_an.pdf

17. Sabharwal S, Kumar A. Methods for Assessing Leg Length Discrepancy. Clin Orthop Relat Res 2008; 466(12):29102922.

18. Rebecca J. Brady, John B. Dean, T. Marc Skinner, Michael T. Gross, Limb Length. Inequality: Clinical Implications for Assessment and Intervention. J Orthop Sports Phys Ther 2003;33(5):221-234.

19. Jackson A S and M. L. Pollock. Alternative Skinfold Measurement Formulas to Calculate Percent Body Fat. Phys Sportsmed 1985; 13:76-90.

20. Franchignoni F, Horak F, Godi M, Nardone A, Giordano A. Using psychometric techniques to improve the Balance Evaluation Systems Test: the mini-BESTest. J Rehabil Med 2010; 42(4):323-31. 
21. Babiarczyk B., Turbiarz A. Body Mass Index in elderly people- do the reference ranges matter? Prog Health Sci 2012; 2(1):58-67.

22. Fjeldstad C, Fjeldstad A S, Acree L C, Nickel K J. and Gardner A W. The influence of obesity on falls and quality of life. Dynamic Medicine 2008; 7(4):1-6.

23. Greve J, Alonso A, Bordini ACPG, Camanho, GL. Correlation between body mass index and postural balance. Clinics 2007;62(6):717-20.

24. Coelho Vilac K H, Oliveira Carneiro J A, Ferriolli E, da Costa Lima N K, Albuquerque de Paula F J, Moriguti J C. Body composition, physical performance and muscle quality of active elderly women. Archives of Gerontology and Geriatrics 2014;59;44-48.

25. Alonso A. C, Luna N. M, Mochizuki L, Barbieri F, Santos S, D'Andreia Greve J. M. The influence of anthropometric factors on postural balance: the relationship between body composition and posturographic measurements in young adults. Clinics 2012;67(12):1433-1441.

26. Del Porto H. C, Pechak C. M, Smith D. R, Reed-Jones R. J. Biomechanical Effects of Obesity on Balance. International Journal of Exercise Science 2013; 5(4): 301-320.

27. Newman A. B, Haggerty C. L, Goodpaster B, Harris T, Kritchevsky S, Nevitt M, Miles T. P, Visser M. Strength and Muscle Quality in a Well-Functioning Cohort of Older Adults: The Health, Aging and Body Composition Study. J Am Geriatr Soc 2003; 51(3):323-330.

28. Mochizuki L, Duarte M, Amadio A. C, Zatsiorsky V. M, Latash M. L. Changes in Postural Sway and Its Fractions in Conditions of Postural Instability. J Appl Biomech. 2006;22(1):51-60.

29. Wang J, Thornton J. C, Kolesnik S, \& Pierson Jr, R. N. .Anthropometry in body composition: an overview. Annals of the New York Academy of Sciences. 2000; 904(1), 317-326. 